\title{
Clinical Perspectives About the Molecular Management of B-Lymphoproliferative Neoplasms
}

\author{
Ibrahim C. HAZNEDAROGLU, Nilgun SAYINALP \\ Hacettepe University Faculty of Medicine, Department of Hematology, Ankara, TURKEY
}

\section{EDITORIAL}

Indolent malignant B-cell lymphoproliferative neoplastic disorders may have a stable disease with only clinical watch and wait approach, or a slowly progressive disease requiring therapy in due course when needed, or a progressive bulky tumor and/or cytopenias representing urgency for chemo-immunotherapy. Therefore, disease presentations in B-lymphoproliferative diseases are heterogeneous and treatment decisions are reached based on the individual patient together with the diverse clinicopathological correlations, staging, risk profile and prognostication. From the clinical point of view, the chemotherapy should not be worse than the disease in B-lymphomas since usually those disorders are indolent and incurable. From the biological point of view, the genesis of the malignant B-cell lymphoproliferative neoplastic disorders is significantly complicated with multi-hit targets. Among the numerous pathobiological pathways, B-cell receptor (BCR) signaling is implicated as a pivotal pathway in tumorigenesis in B-cell malignancies. Moreover, the mechanisms of the BCR activation are quite diverse and range from chronic antigenic drive by microbial or viral antigens to auto-stimulation of B-cells by self-antigens to activating mutations in intracellular components of the BCR pathway. ${ }^{1}$ Signaling activation via the BCR could drive neoplastic cell proliferation and survival via the cascade of involved and upregulated protein kinases. Thus, understanding the biology of the BCR-signaling pathway has led to identification of novel molecular targets and the development of drugs capable of targeting pathways downstream of the BCR. Particularly the inhibitors of Bruton's tyrosine kinase (BTK) and phosphatidylinositide 3-kinase-delta have included into the clinical studies and demonstrated high response rates in B-cell lymphoproliferative neoplastic disorders such as chronic lymphocytic leukemia (CLL). ${ }^{2}$ Registered clinical trials of Ibrutinib, a small molecule that irreversibly inhibits BTK, are depicted in Table 1.
CLL represents a unique clinical model to test the efficacy of BCR modulation via BTK inhibition. Therefore most of the clinical studies with ibrutinib are being performed in the field of CLL. The imbalance of the biological events particularly proliferation and apoptosis, is the novel target in the treatment of CLL. The newly developed targeted molecular agents such as ibrutinib (PCI-32765), idelalisib (CAL-101), Navitoclax (ABT263), and ABT-199 try to balance between survival and programmed cell death in the pathobiology of the disease. $^{3}$

Clinical development of the targeted molecules such as Ibrutinib, inhibiting intracellular pathways that promote the proliferation and survival of malignant Blymphocytes may lead to a chemotherapy-free area for the patients. ${ }^{4}$ Meanwhile, the mutations resulting in the resistance to the drugs $^{5}$, disease recurrences following partial responses, the cost, and specific adverse events (coagulopathy of ibrutinib, hepatotoxicity of idelalisib) 4 will be the next challenging areas. It is hoped that the ongoing clinical trials focusing on the combinations of kinase inhibitors with monoclonal antibodies, chemotherapeutics and other pro-apoptotic agents will be helpful for better management of the patients with malignant B-cell lymphoproliferative neoplastic diseases.

\section{REFERENCES}

1. Niemann CU, Wiestner A. B-cell receptor signalling as a driver of lymphoma development and evolution. Semin Cancer Biol 23: 410-421, 2013.

2. Danilov AV. Targeted Therapy in Chronic Lymphocytic Leukemia: Past, Present, and Future. Clin Therapeutics 35: 12581270, 2013.

3. Hallek M. A chemotheraphy-free future? Novel treatment strategies for chronic lymphocytic leukemia. Hematol Education 8: 75-86, 2014.

4. Cheson BD. CLL and NHL: the end of chemotherapy? Blood 123: 3368-70, 2014.

5. Cheng S, Lu P, Setty M, et al. A Novel Mutation in Bruton Tyrosine Kinase Confers Acquired Resistance to Ibrutinib (PCl-32765) in Chronic Lymphocytic Leukemia. J Molecular Diagnostics 15: 859A , 2013. 
Table 1. Registered clinical trials of Ibrutinib (completed, recruiting, or not yet recruiting in www.clinicaltrials.gov)

\section{STUDY AREA}

Clinical studies in Chronic Lymphocytic Leukemia

Clinical Studies inFollicular Lymphoma

Clinical Studies in Mantle Cell Lymphoma

\section{STUDY TITLE}

- $\quad$ Ibrutinib Versus Ibrutinib + Rituximab (i vs iR) in Patients with Relapsed Chronic Lymphocytic Leukemia (CLL)

- $\quad$ PCl-32765 (Ibrutinib) in Treating Patients with Relapsed or Refractory Chronic Lymphocytic Leukemia, Small Lymphocytic Lymphoma, or B-cell Prolymphocytic Leukemia

- Rituximab and Bendamustine Hydrochloride, Rituximab and Ibrutinib, or Ibrutinib Alone in Treating Older Patients with Previously Untreated Chronic Lymphocytic Leukemia

- Lenalidomide and Ibrutinib in Treating Patients with Relapsed or Refractory Chronic Lymphocytic Leukemia or Small Lymphocytic Lymphoma

- Lenalidomide, Ibrutinib, and Rituximab in Treating Patients with Relapsed or Refractory Chronic Lymphocytic Leukemia or Small Lymphocytic Lymphoma

- Ibrutinib and Rituximab Compared with Fludarabine Phosphate, Cyclophosphamide, and Rituximab in Treating Patients with Untreated Chronic Lymphocytic Leukemia or Small Lymphocytic Lymphoma

- A Study of PCl-32765 (Ibrutinib) Versus Rituximab in Relapsed or Refractory Chronic Leukemia/Lymphoma

- Pilot Study to Determine Effects of the Btk Inhibitor PCl-32765 on Leukemia Cell Kinetics and Trafficking, Using Heavy Water Labeling in Subjects with CLL and SLL

- A Study of Ibrutinib in Combination with Bendamustine and Rituximab in Patients with Relapsed or Refractory Chronic Lymphocytic Leukemia or Small Lymphocytic Lymphoma

- A Phase 3 Study of Ibrutinib (PCl-32765) Versus Ofatumumab in Patients with Relapsed or Refractory Chronic Lymphocytic Leukemia

- A Multicenter Phase 2 Study of PCl-32765 (lbrutinib) in Patients with Relapsed or Refractory Chronic Lymphocytic Leukemia (CLL) or Small Lymphocytic Lymphoma (SLL) with $17 p$ Deletion

- $\quad$ PCl-32765 for Special Cases of Chronic Lymphocytic Leukemia or Small Lymphocytic Lymphoma

- $\quad$ Phase 2 Study of the Combination of Bruton's Tyrosine Kinase Inhibitor PCl-32765 and Rituximab in High-Risk Chronic Lymphocytic Leukemia and Small Lymphocytic Lymphoma Patients

- A Multicenter, Open-label, Phase 3 Study of the Bruton's Tyrosine Kinase Inhibitor PCl-32765 Versus Chlorambucil in Patients 65 Years or Older with Treatment-naive Chronic Lymphocytic Leukemia or Small Lymphocytic Lymphoma (RESONATETM-2)

- Safety and Tolerability Study of PCl-32765 Combined with Fludarabine/Cyclophosphamide/ Rituximab (FCR) and Bendamustine/Rituximab (BR) in Chronic Lymphocytic Leukemia (CLL)

- A Phase 2 Open-Label Study of the Efficacy and Safety of ABT-199 (GDC-0199) in Chronic Lymphocytic Leukemia (CLL) Subjects with Relapse or Refractory to B-Cell Receptor Signaling Pathway Inhibitor Therapy

- A Study of PCl-32765 (Ibrutinib) in Patients with Refractory Follicular Lymphoma

- Rituximab, Lenalidomide, and Ibrutinib in Treating Patients with Previously Untreated Stage II-IV Follicular Lymphoma

- Ibrutinib in Treating Patients with Relapsed or Refractory Follicular Lymphoma

- Study of the Bruton's Tyrosine Kinase Inhibitor in Combination with Rituximab in Previously Untreated Subjects with Follicular Lymphoma

- Study of the Bruton's Tyrosine Kinase (BTK) Inhibitor Ibrutinib in Participants with cell lymphoma Relapsed or Refractory Mantle Cell Lymphoma

- Ibrutinib and Palbociclib Isethionate in Treating Patients with Previously Treated Mantle Cell Lymphoma

- An Open Label Treatment Use Protocol for Ibrutinib in Subjects with Relapsed or Refractory

- A Study to Evaluate the Efficacy and Safety of Ibrutinib, in Patients with Mantl Cell Lymphoma Who Progress After Bortezomib Therapy

- Study of Ibrutinib (a Bruton's Tyrosine Kinase Inhibitor), Versus Temsirolimus in Patients with Relapsed or Refractory Mantle Cell Lymphoma Who Have Received at Least One Prior Therapy 
Table 1. (continue) Registered clinical trials of Ibrutinib (completed, recruiting, or not yet recruiting in www.clinicaltrials. gov)

STUDY AREA

Clinical Studies in Diffuse Large B-cell lymphoma

Clinical studies in B-Cell non-Hodgkin Lymphomas

Clinical Studies in Marginal Zone Lymphoma

Clinical studies in Hairy Cell Leukemia

Clinical Studies in Plasma Cell Disorders

Clinical Studies in Acute Lymphoblastic Leukemia

\section{STUDY TITLE}

- Phase 2 Ibrutinib + Rituximab in Relapsed/Refractory Mantle Cell Lymphoma (R/R MCL)

- A Study of the Bruton's Tyrosine Kinase Inhibitor Ibrutinib Given in Combination with Bendamustine and Rituximab in Patients with Newly Diagnosed Mantle Cell Lymphoma

- Safety and Efficacy of PCl-32765 in Subjects with Relapsed/Refractory Mantle Cell Lymphoma (MCL)

- A Study of the Bruton's Tyrosine Kinase Inhibitor, PCl-32765 (Ibrutinib), in Combination with Rituximab, Cyclophosphamide, Doxorubicin, Vincristine, and Prednisone in Patients with Newly Diagnosed Non-Germinal Center B-Cell Subtype of Diffuse Large B-Cell Lymphoma

- Ibrutinib in Combination with Lenalidomide, with and without Rituximab in Participants with Relapsed or Refractory Diffuse Large B-Cell Lymphoma

- Rituxan/Bendamustine/PCl-32765 in Relapsed DLBCL, MCL, or Indolent NonHodgkin's Lymphoma

- Ibrutinib and Lenalidomide with Dose Adjusted EPOCH-R in Subjects with Relapsed/Refractory Diffuse Large B-cell Lymphoma

- Safety and Efficacy Study of Bruton's Tyrosine Kinase Inhibitor in Subjects with Relapsed or Refractory Diffuse Large B-cell Lymphoma

- A Study Combining Ibrutinib with Rituximab, Cyclophosphamide, Doxorubicin, Vincristine, and Prednisone in Patients with CD20-Positive B-Cell Non Hodgkin Lymphoma

- Ibrutinib in Treating Relapsed or Refractory B-Cell Non-Hodgkin Lymphoma in Patients with HIV Infection

- Ublituximab + Ibrutinib in Select B-cell Malignancies

- A Study of PCl-32765 (Ibrutinib) in Combination with Either Bendamustine and Rituximab or Rituximab, Cyclophosphamide, Doxorubicin, Vincristine, and Prednisone in Patients with Previously Treated Indolent Non-Hodgkin Lymphoma

- Lenalidomide and Ibrutinib in Treating Patients with Relapsed or Refractory B-Cell Non-Hodgkin Lymphoma

- Bruton's Tyrosine Kinase (BTK) Inhibition in B-cell Lymphomas

- A Study to Evaluate the Bruton's Tyrosine Kinase (Btk) Inhibitor PCl-32765 in Patients with Recurrent Mature B-Cell Neoplasms

- Study of the Bruton's Tyrosine Kinase Inhibitor in Subjects with Relapsed/Refractory Marginal Zone Lymphoma

- Ibrutinib in Treating Patients with Relapsed Hairy Cell Leukemia

- Study of the Bruton's Tyrosine Kinase Inhibitor in Combination with Carfilzomib cell diseases (Kyprolis ${ }^{\mathrm{TM}}$ ), in Subjects with Relapsed or Relapsed and Refractory Multiple Myeloma

- Study of the Bruton's Tyrosine Kinase Inhibitor in Subjects with Relapsed or Relapsed and Refractory Multiple Myeloma

- Ibrutinib (PCl-32765) in Waldenstrom's Macroglobulinemia

- Ibrutinib with Rituximab in Previously Treated Adults with Waldenstrom's Macroglobulinemia

- Ibrutinib in Treating Patients with Relapsed or Refractory B-cell Acute Lymphoblastic Leukemia

\section{Correspondence}

Dr. İbrahim C. HAZNEDAROĞLU

Hacettepe Üniversitesi Tıp Fakültesi

İç Hastalıkları Anabilim Dalı

Hematoloji Bilim Dalı

Sihhiye, ANKARA / TURKEY
Tel: (+90.312) 3051543

Fax: (+90.312) 3051614

e-mail: ichaznedaroglu@gmail.com 